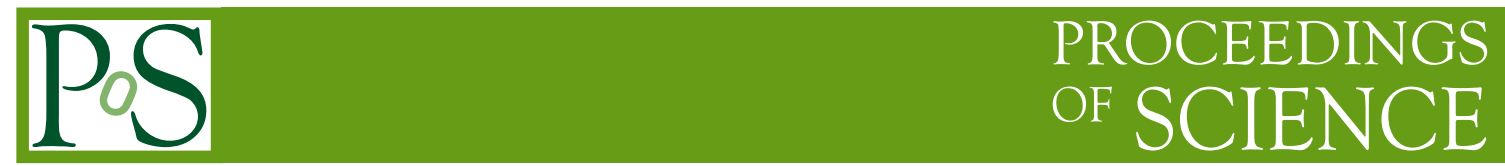

\title{
Supersymmetry Searches at ATLAS
}

\author{
M. Fehling-Kaschek ${ }^{* \dagger}$ \\ Albert-Ludwigs Universität Freiburg \\ E-mail: mirjam.fehling@physik.uni-freiburg.de
}

Searches for Supersymmetry with the ATLAS dectector are presented for integrated luminosities up to $2 \mathrm{fb}^{-1}$ of $p p$ collisions at $\sqrt{s}=7 \mathrm{TeV}$. A large variety of models is tested and limits are shown for miscellaneous parameter regions.

LHC on the March,

November 16-18, 2011

Protvino, Moscow region, Russian Federation

* Speaker.

${ }^{\dagger}$ On behalf of the ATLAS Collaboration 


\section{Introduction}

Supersymmetry (SUSY) [1] is one of the most theoretically promising candidates to solve some of the open questions of the Standard Model (SM). In its simplest form, every Standard Model particle has a superpartner which differs by half a unit of spin and that should have a mass not far from the $\mathrm{TeV}$ scale.

The general minimal extension of the SM (MSSM) has more than 100 free parameters. This number is usually reduced by further assumptions to a few free parameters. These constrained models are used first to optimise the analyses and second to derive limits, once the data has been analysed. Two different types of models are used: first, models where the SUSY breaking is induced by the interaction with a hidden sector [2]. Parameter reduction is achieved by assuming unification at the GUT scale. Examples are the minimal supergravity (mSUGRA) and the gauge mediated SUSY breaking (GMSB). Second, simplified or phenomenological MSSM models are used. Here the masses and hierarchy of the SUSY particles are set to specific values and only one or two dedicated decay chains of the primarily produced SUSY particles play a role.

In order to suppress the violation of lepton and baryon numbers of the general MSSM lagrangian, the conservation of the so called $R$-parity is imposed. In $R$-parity conserving models, SUSY particles are produced in pairs and the lightest supersymmetric particle (LSP) is stable. The LSP is a good candidate for dark matter and leads to missing transverse momentum as characteristic signature for the analyses. The first part of this contribution will cover those signatures (section 2). The second part (section 3 ) covers $R$-parity violating searches, either looking for a resonance in the $e \mu$-mass spectrum or looking for a massive displaced vertex.

The amount of analysed data ranges from an integrated luminosity $\mathscr{L}=33 \mathrm{pb}^{-1}$ to $\mathscr{L}=2 \mathrm{fb}^{-1}$ and was taken at a center of mass energy of $\sqrt{s}=7 \mathrm{TeV}$. Most of the results are shown for $\mathscr{L} \sim 1 \mathrm{fb}^{-1}$, collected by the ATLAS detector [3] until summer 2011.

\section{2. $E_{\mathrm{T}}^{\text {miss }}$ based searches}

In $R$-parity conserving models the SUSY particles are produced in pairs and decay through rather long decay chains into the LSP which is stable and leaves the detector undetected, leading to a high missing transverse momentum (whose magnitude is denoted by $E_{\mathrm{T}}^{\text {miss }}$ ). A typical SUSY event is sketched in Fig. 1. Different final states are defined to cover the variety of SUSY parameter space regions, depending on the mass spectrum and decay modes of the SUSY particles:

- 0-lepton: strong gluino $(\tilde{g})$ or squark $(\tilde{q})$ production

- multijets: $\tilde{g} \tilde{g}$ production with long decay chains

- 1-lepton: leptons produced in the decay chains of $\tilde{g} / \tilde{q}$ via a slepton $(\tilde{l})$ or $W$

- 2-lepton: like 1-lepton plus direct gaugino production

- b-jets: direct or $\tilde{g}$-mediated production of sbottoms $(\tilde{b})$ or stops $(\tilde{t})$

The main backgrounds are SM processes with either real $E_{\mathrm{T}}^{\text {miss }}$ due to neutrinos $(t \bar{t}, W+\mathrm{jets}$, $Z+$ jets) or fake $E_{\mathrm{T}}^{\text {miss }}$ from mismeasured jets (QCD multijet events). They are mostly estimated 


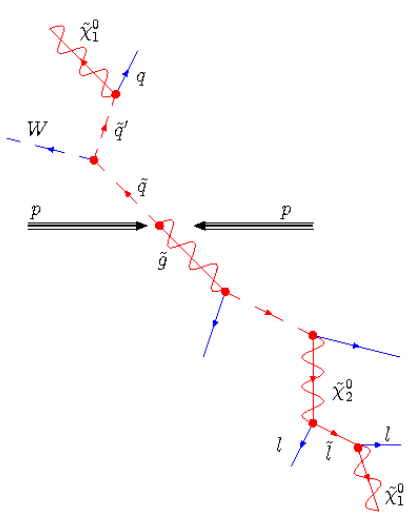

Figure 1: A typical $R$-parity conserving SUSY event: Inicially a pair of sparticles is produced, which eventually decays into two LSPs.

with data-driven methods. Pure Monte Carlo (MC) predictions are used for minor backgrounds. For the data-driven estimates control regions (CR) are defined to normalise the background. The event yield in the signal region (SR) is then obtained by a transfer factor, taken either from MC or directly from data: $N_{S R}=T F \cdot N_{C R}$.

\subsection{0-lepton analysis}

The 0-lepton analysis [4] aims at the strong production of squarks and gluinos decaying to the LSP. Five signal regions with high $E_{\mathrm{T}}^{\mathrm{miss}}(130 \mathrm{GeV})$, at least 2, 3 or 4 jets with different $p_{\mathrm{T}}$ threshold $\left(p_{\mathrm{T}}(\right.$ jet1 $\left.)>130 \mathrm{GeV}\right)$ and no lepton are used to maximise the sensitivity for different models. The scalar sum of $E_{\mathrm{T}}^{\text {miss }}$ and the transverse momentum of the jets, the effective mass $\mathrm{m}_{\mathrm{eff}}$, is used to discriminate the possible SUSY signal from the background.

The main SM background processes are $W \rightarrow \tau v, Z \rightarrow v v$ and hadronic tau decays in top events. The multijet QCD background is largely reduced by rejecting events where the direction of the jets is close to the direction of $E_{\mathrm{T}}^{\text {miss }}$. For each background a dedicated CR is defined and then a combined likelihood fit of all CRs is performed.

The $\mathrm{m}_{\mathrm{eff}}$ distributions for all signal regions are shown in Fig. 2 after the event selection. The arrows indicate where the final cut on $\mathrm{m}_{\mathrm{eff}}$ is applied. As no excess over the SM expectation is observed, exclusion limits are derived for several SUSY models. Two examples are shown in Fig. 3. For the left plot a phenomenological MSSM model is assumed, where the neutralino $\left(\tilde{\chi}_{1}^{0}\right)$ is the LSP and only gluinos and first two generation squarks play a role (for other SUSY particles the mass is set to $5 \mathrm{TeV}$ ) [5]. The limit is computed for three different neutralino masses $\left(m_{\tilde{\chi}_{1}^{0}}=0,195,395 \mathrm{GeV}\right)$. Masses up to $1075 \mathrm{GeV}$ can be excluded for equal squark and gluino masses. The limit obtained for an mSUGRA model with $\tan \beta=10, A_{0}=0$ and $\mu>0$ is shown in the right plot as a function of $m_{0}$ and $m_{1 / 2}$. For this model masses up to $980 \mathrm{GeV}$ can be excluded for $m_{\tilde{g}}=m_{\tilde{q}}$.

\subsection{Multijets analysis}

The multijet search [6] focuses on long decay chains of gluinos. Compared to the 0-lepton 

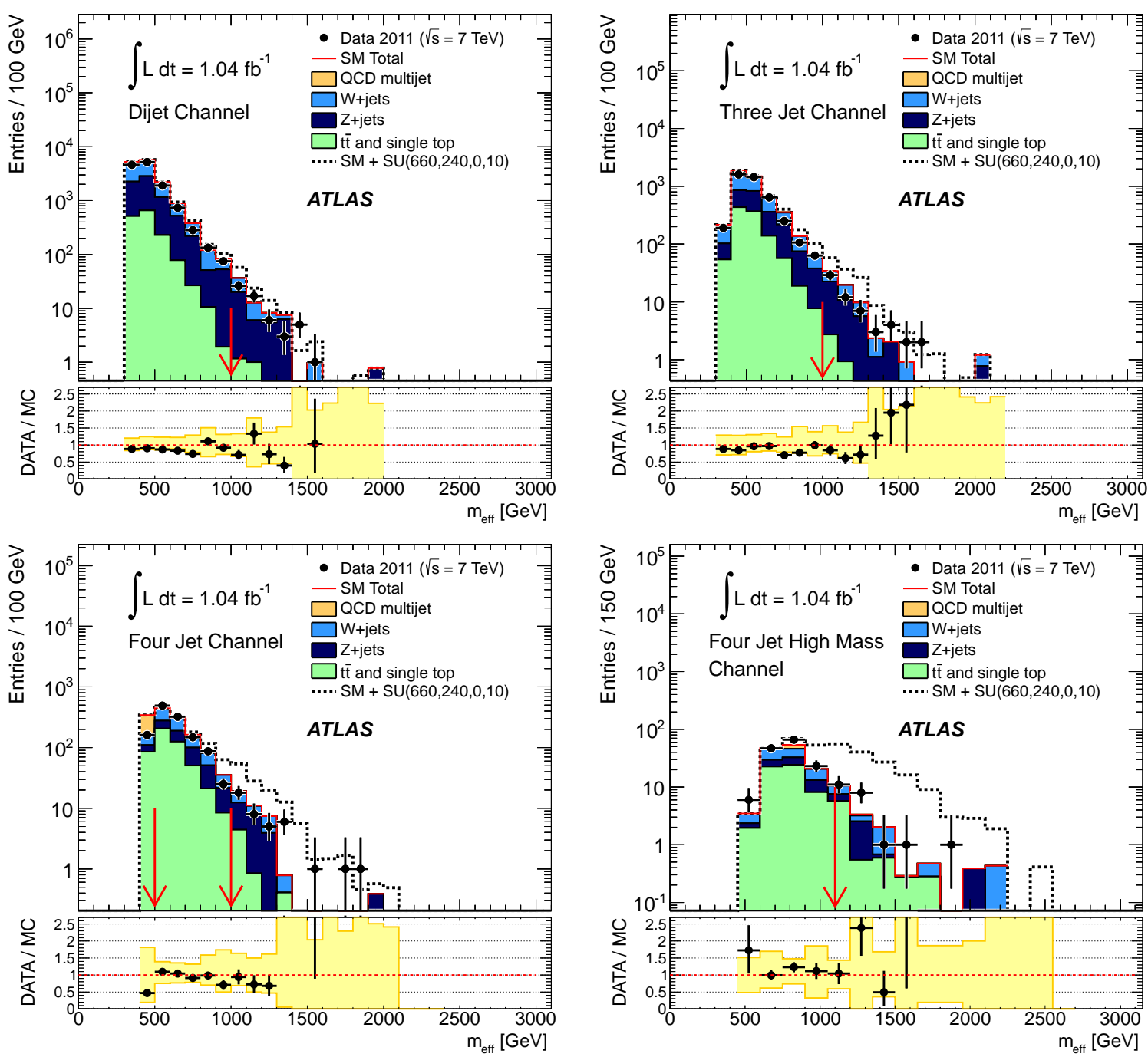

Figure 2: $\mathrm{m}_{\mathrm{eff}}$ distributions for the 0-lepton analysis: $\geq 2$ jets (top left), $\geq 3$ jets (top right), $\geq 4$ jets with $p_{\mathrm{T}}\left(\right.$ jet $\left._{2.4}\right)>40 \mathrm{GeV}$ (bottom left), $\geq 4$ jets with $p_{\mathrm{T}}\left(\right.$ jet $\left._{2 . .4}\right)>80 \mathrm{GeV}$ (bottom right). The final cuts on $\mathrm{m}_{\mathrm{eff}}$ are indicated by the red arrows. The dashed lines are showing a reference mSUGRA signal point $\left(m_{0}=660 \mathrm{GeV}, m_{1 / 2}=240 \mathrm{GeV}, \tan \beta=10, A_{0}=0\right)$.

analysis events with lower $E_{\mathrm{T}}^{\text {miss }}$ but harsher jet requirements are selected. The four signal regions are defined by at least 6,7 or 8 jets and no leptons. As discriminating variable the ratio of $E_{\mathrm{T}}^{\text {miss }}$ and $\sqrt{\sum p_{\mathrm{T}}(\mathrm{jets})}$ is taken. This variable is found to be invariant under the jet multiplicity variation and is used to estimate the main background arising from QCD multijet production from control regions with lower jet multiplicities.

In Fig. 4 the exclusion limit is shown for the same mSUGRA scenario as shown for the 0-lepton search. Gluino masses up to $520 \mathrm{GeV}$ can be excluded.

\subsection{1-lepton analysis}

Leptons can be produced if $W$ or $Z$-bosons or sleptons occur in the decay chains of the squarks or gluinos. Four signal regions with either an electron or a muon are used in the 1-lepton analysis [7]. 

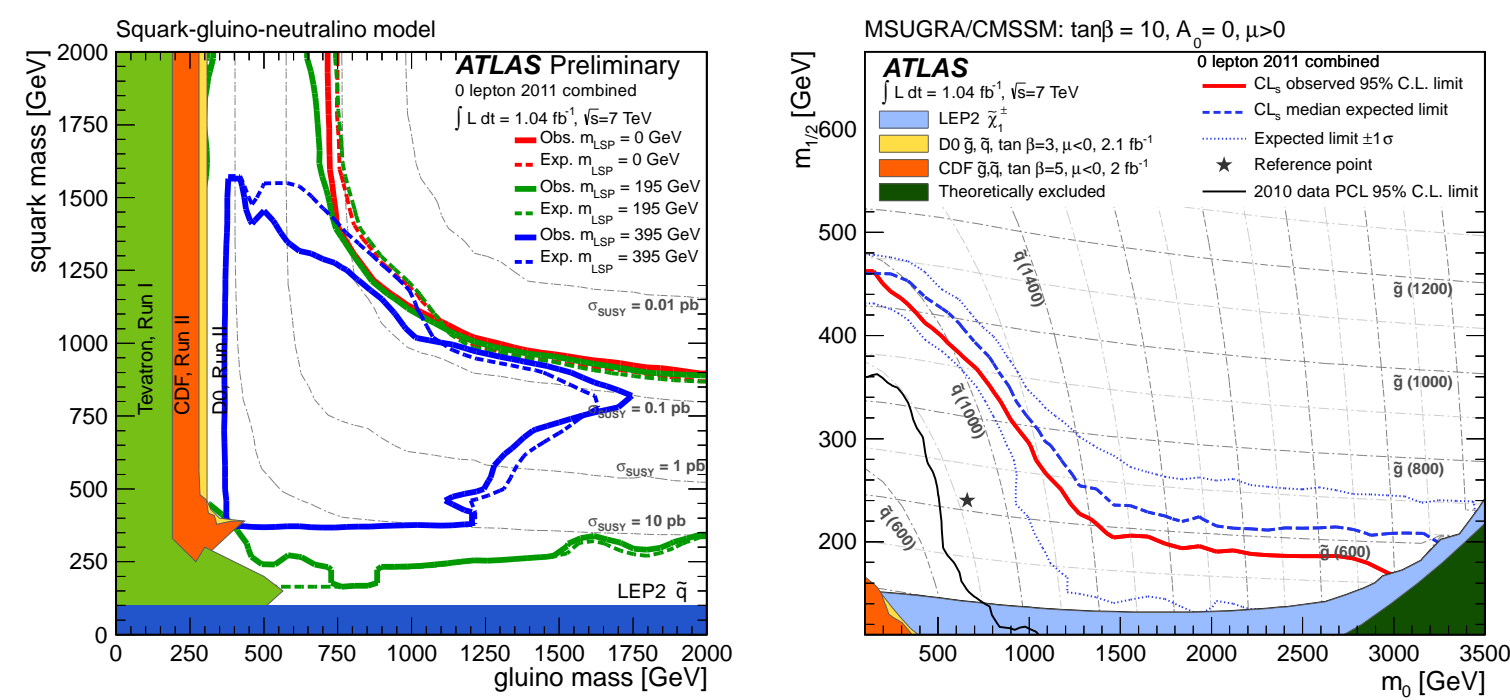

Figure 3: Exclusion limits of the 0-lepton analysis: The left plot shows the exclusion in the context of a phenomenological model in the squark-gluino mass plane for different masses of the LSP $\left(m_{\tilde{\chi}_{1}^{0}}=0,195\right.$, $395 \mathrm{GeV})$. On the right plot the exclusion limit is shown in the $m_{0}-m_{1 / 2}$ plane of the mSUGRA model $\left(\tan \beta=10, A_{0}=0, \mu>0\right)$.

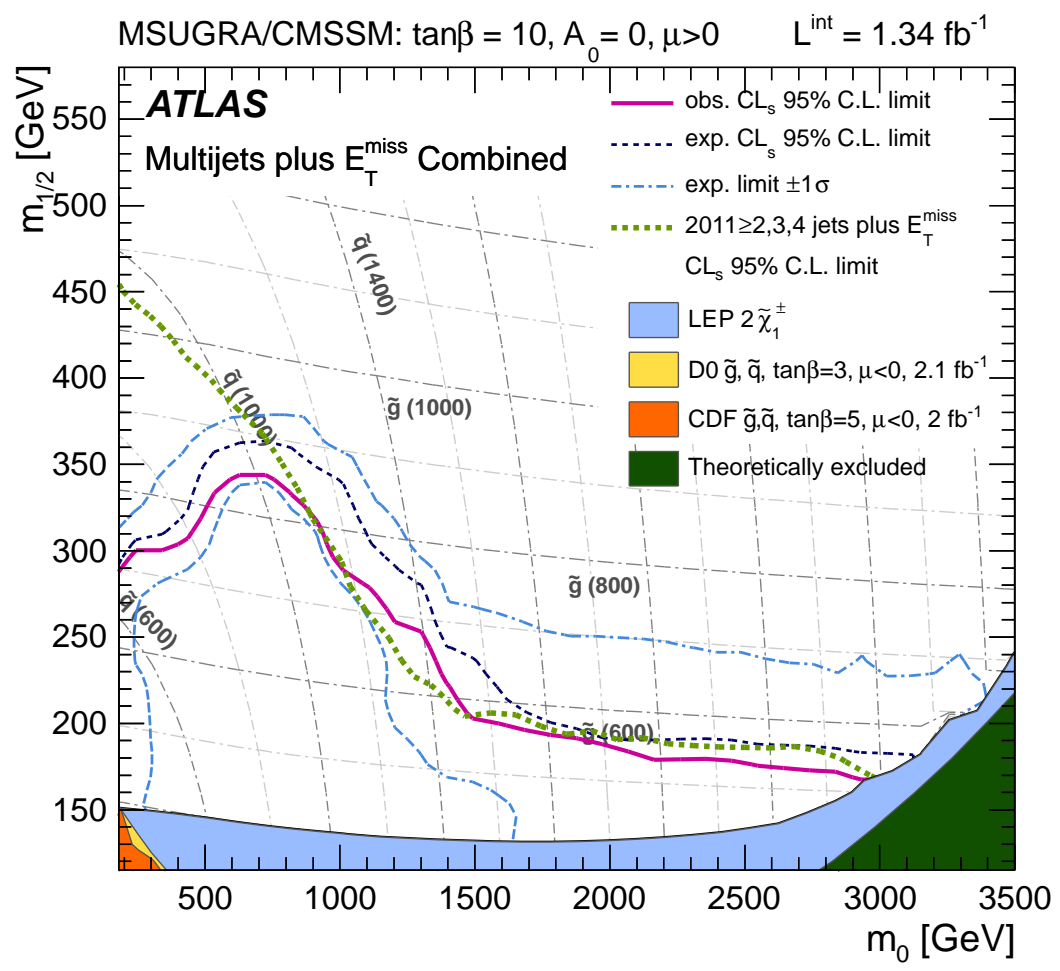

Figure 4: Exclusion limit of the multijet analysis in the $m_{0}-m_{1 / 2}$ plane of the mSUGRA model $(\tan \beta=10$, $A_{0}=0, \mu>0$ ). The result of the 0 -lepton analysis is shown as reference in green. 


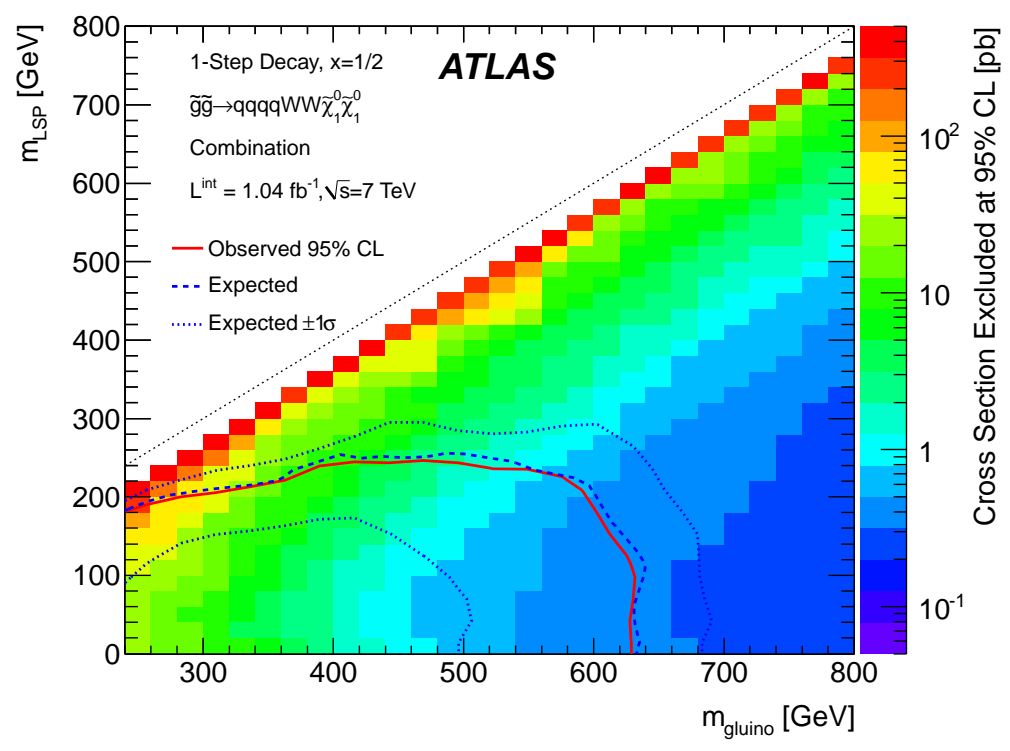

Figure 5: Exclusion limit of the 1-lepton analysis for a simplified model. Gluinos are pair-produced and then decay to the LSP via a chargino. For each point the model-independent limit of the cross-section is also shown, represented by the color-code.

In order to suppress $E_{\mathrm{T}}^{\text {miss }}$ contributions from $W \rightarrow l v$, the tranverse mass formed by the lepton and $E_{\mathrm{T}}^{\text {miss }}$ is employed. In addition the effective mass is used as discriminating variable. Here also the lepton $p_{\mathrm{T}}$ is added in addition to $E_{\mathrm{T}}^{\text {miss }}$ and the jets' momentum.

As no excess over the SM expectation is observed, limits are calculated for differenct simplified MSSM models. One example is shown in Fig. 5 for gluino pair production with one step decays of the gluinos to the LSP via charginos. Gluino masses up to $600 \mathrm{GeV}$ can be excluded for LSP masses up to $200 \mathrm{GeV}$.

\subsection{2-lepton analysis}

Similar to the 1-lepton channel, the 2-lepton analysis [8] is looking for leptons produced in the decay chains of gluinos and squarks. In addition this channel is also sensitive to models with direct gaugino production.

Different signal regions based on $E_{\mathrm{T}}^{\mathrm{miss}}$ and the jet multiplicity and momentum have been defined, with either same sign or opposite sign leptons. While dileptonic $t \bar{t}$ is the main background for the opposite sign analysis, the background of the same sign analysis is dominated by diboson and charge mismeasured events.

In all signal regions a good agreement between data and SM prediction is found. An interpretation of the results is made in the context of a GMSB scenario [9]. In Fig. 6 the exclusion limit is shown in the $\Lambda$ - $\tan \beta$ plane for the opposite sign analysis.

\subsection{B-jet analysis}

For specific models, light third generation squarks are favoured, because the possible mixing 


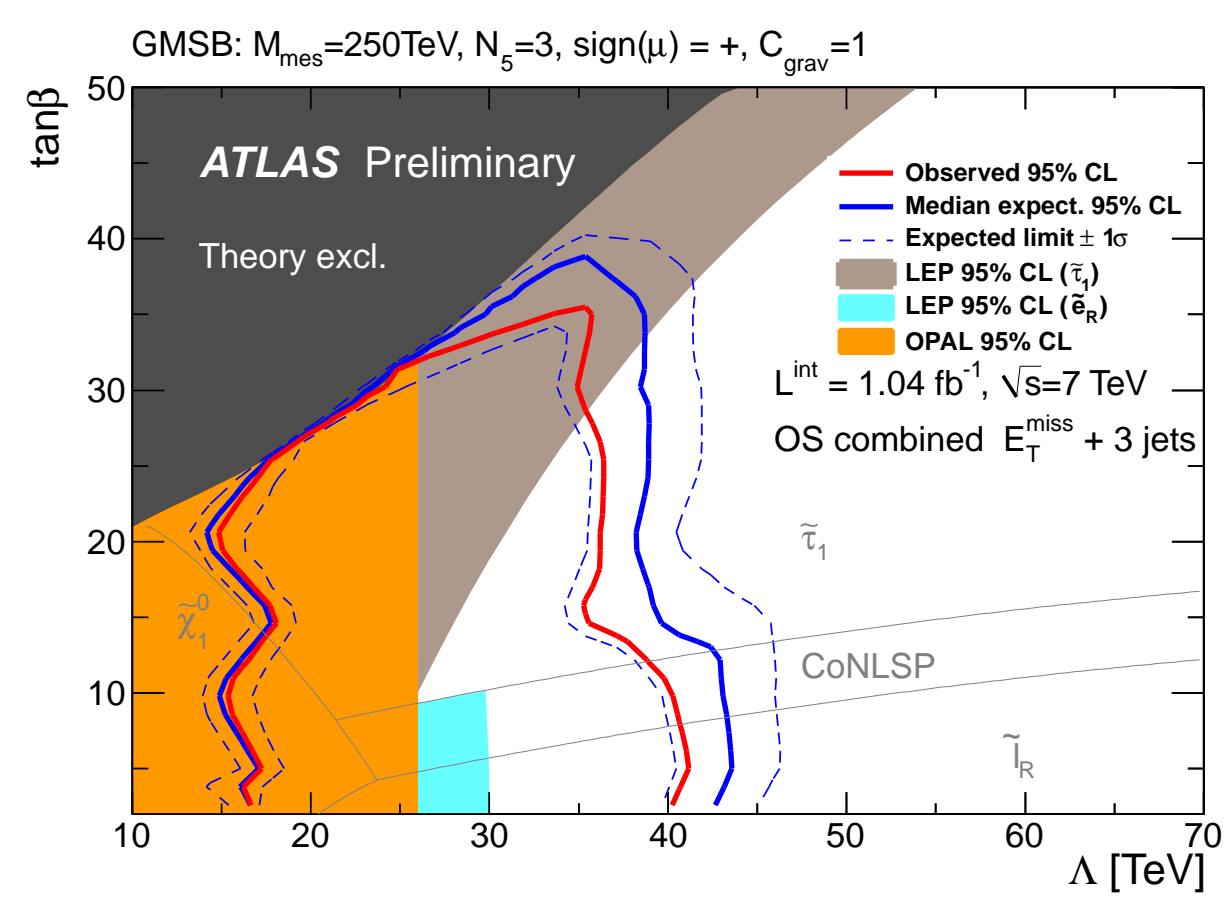

Figure 6: Exclusion limit of the 2-lepton analysis with opposite sign leptons in the $\Lambda$-tan $\beta$ plane of a GMSB model $\left(M_{\text {mes }}=250 \mathrm{TeV}, N_{5}=3, \mu>0, C_{\text {grav }}=1\right)$. The next to lightest SUSY-particles are either the $\tilde{\chi}_{1}^{0}$, the $\tilde{\tau}_{1}$ or the $\tilde{l}_{R}$ as marked in the plot (in the CoNLSP region the $\tilde{\tau}_{1}$ and $\tilde{l}_{R}$ are degenerate).

of mass and weak eigenstates is proportional to the fermion mass. These analyses are looking for $b$-jets as the sbottom or stop decay usually results in a $b$-quark. Sbottoms or stops can be produced either via gluino decay (gluino mediated production) or in a direct pair production.

For the gluino mediated sbottom and stop production two channels have been studied. One with no leptons [10], where four signal regions with either one or two $b$-jets and high $E_{\mathrm{T}}^{\text {miss }}(>130 \mathrm{GeV}$ ) are defined, and one with an electron or muon [11], where one signal region with one $b$-jet, a lower $E_{\mathrm{T}}^{\text {miss }}$ threshold $(>80 \mathrm{GeV})$ and a cut on the tranverse mass of the lepton and $E_{\mathrm{T}}^{\text {miss }}$ is used. Both analyses use the effective mass as discriminating variable. The main background production process is $t \bar{t}$, followed by $W+$ (heavy flavour)jets.

A very good agreement for data and expected number of SM events is found for the no lepton case. The exclusion limits for two different simplified models is shown in Fig. 7. On the left plot the limit is shown in the gluino-sbottom mass plane. The underlying model is assuming the following decay chain for each of the pair produced gluinos: $\tilde{g} \rightarrow \tilde{b}_{1} b \rightarrow b b \tilde{\chi}_{1}^{0}$, where the $\tilde{\chi}_{1}^{0}$-mass is $60 \mathrm{GeV}$. For this model gluino masses up to $720 \mathrm{GeV}$ are excluded for sbottom masses below $600 \mathrm{GeV}$. The plot on the right is showing the limit in the gluino-neutralino mass plane for a model considering the decay $\tilde{g} \rightarrow b b \tilde{\chi}_{1}^{0}$ via an off-shell sbottom. Gluino masses below $660 \mathrm{GeV}$ are excluded for neutralino masses below $200 \mathrm{GeV}$.

For the 1-lepton analysis similar models are tested, replacing the sbottoms by stops and $b$-quarks by $t$-quarks. The additional lepton appears in the top decay. The exclusion limits are shown in Fig. 8. The observed limits are visibly below the expected limits, which is due to a non-significant 

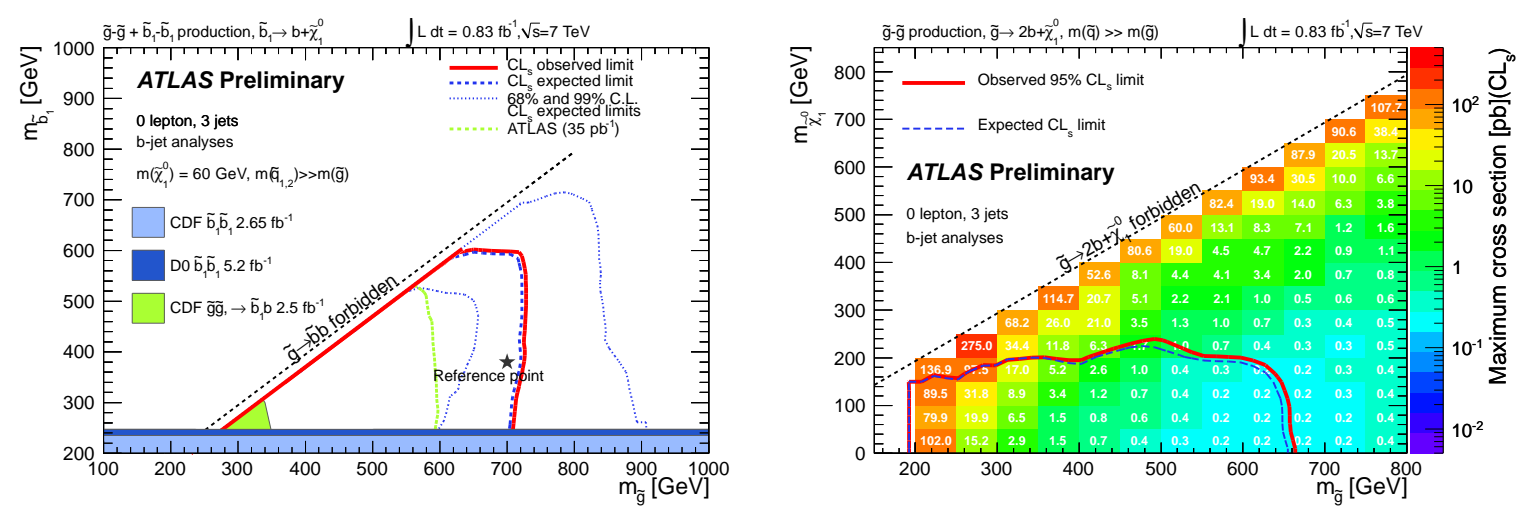

Figure 7: Exclusion limits of the $b$-jets with no lepton analysis for simplified models. $\tilde{g} \tilde{g}$ production with $\tilde{g} \rightarrow \tilde{b}_{1} b \rightarrow b b \tilde{\chi}_{1}^{0}$ (left) and $\tilde{g} \rightarrow b b \tilde{\chi}_{1}^{0}$ via an off-shell sbottom (right) is assumed. For the right plot also the limit on the cross-section for each signal point is shown (color-code).
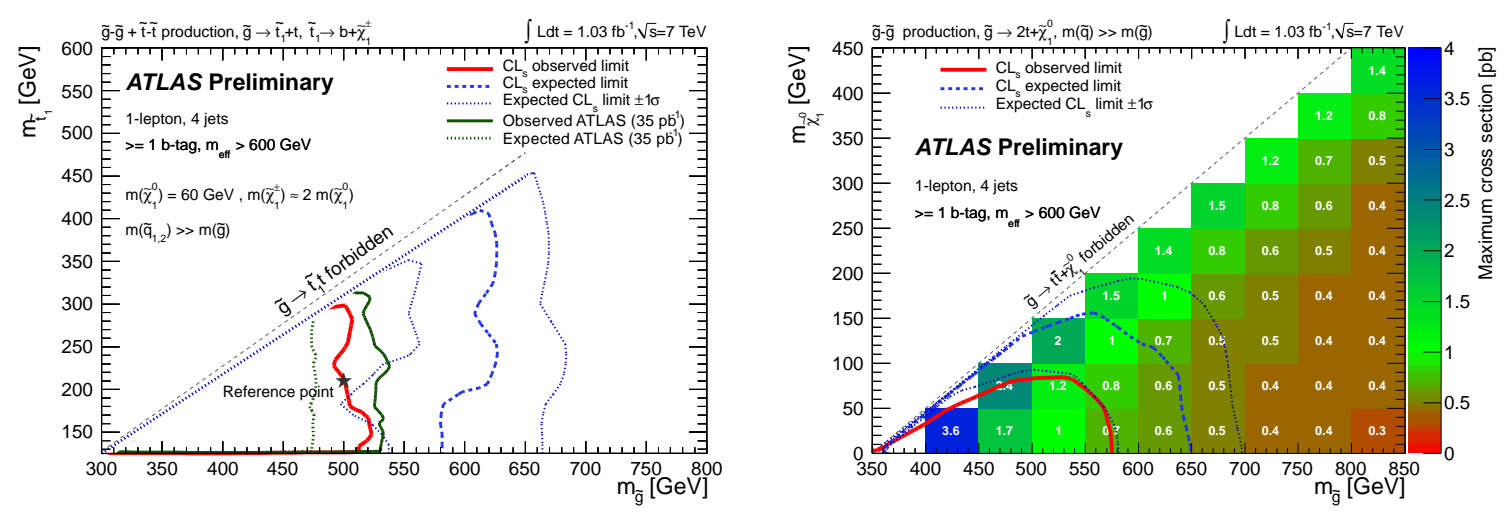

Figure 8: Exclusion limits of the $b$-jets with 1-lepton analysis for simplified models. $\tilde{g} \tilde{g}$ production with $\tilde{g} \rightarrow \tilde{t}_{1} t \rightarrow b t \tilde{\chi}^{ \pm}$(left) and $\tilde{g} \rightarrow t t \tilde{\chi}_{1}^{0}$ via an off-shell stop (right) is assumed, where $m_{\tilde{\chi}_{1}^{0}}=60 \mathrm{GeV}=0.5 \cdot m_{\tilde{\chi}}^{ \pm}$. For the right plot also the limit on the cross-section for each signal point is shown (color-code).

upward fluctuation of data events compared to the expected SM events (74 data events, compared to $54.9 \pm 13.6$ expected SM events). Gluino masses up to $520 \mathrm{GeV}$ can be excluded for stop masses up to $300 \mathrm{GeV}$ for the model assuming $\tilde{g} \tilde{g}$ production with the $\tilde{g} \rightarrow \tilde{t}_{1} t \rightarrow b t \tilde{\chi}^{ \pm}$decay. Gluino masses below $570 \mathrm{GeV}$ are excluded for neutralino masses below $40 \mathrm{GeV}$ for the model assuming the $\tilde{g} \rightarrow t t \tilde{\chi}_{1}^{0}$ decay via an off-shell stop.

The analysis looking for the direct production of sbottom pairs [12] is based on the selection of events with two $b$-jets $\left(p_{\mathrm{T}}>130,50 \mathrm{GeV}\right)$ and high $E_{\mathrm{T}}^{\text {miss }}(>130 \mathrm{GeV})$. As final discriminating variable the boost-corrected contransverse mass $\mathrm{m}_{\mathrm{CT}}$ [13] of the two $b$-jets is used. Three signal regions are defined with $\mathrm{m}_{\mathrm{CT}}>100,150$ and $200 \mathrm{GeV}$. The $\mathrm{m}_{\mathrm{CT}}$ and $E_{\mathrm{T}}^{\text {miss }}$ distribution are shown in Fig. 9. The number of observed data events is in good agreement with the SM expectation for all signal regions. An exclusion limit is calculated for a simplified model, where both pair produced sbottoms decay to $b+\tilde{\chi}_{1}^{0}$ and is shown in Fig. 10. Sbottoms masses up to $350 \mathrm{GeV}$ for $m_{\tilde{\chi}_{1}^{0}}<600 \mathrm{GeV}$ are excluded. 


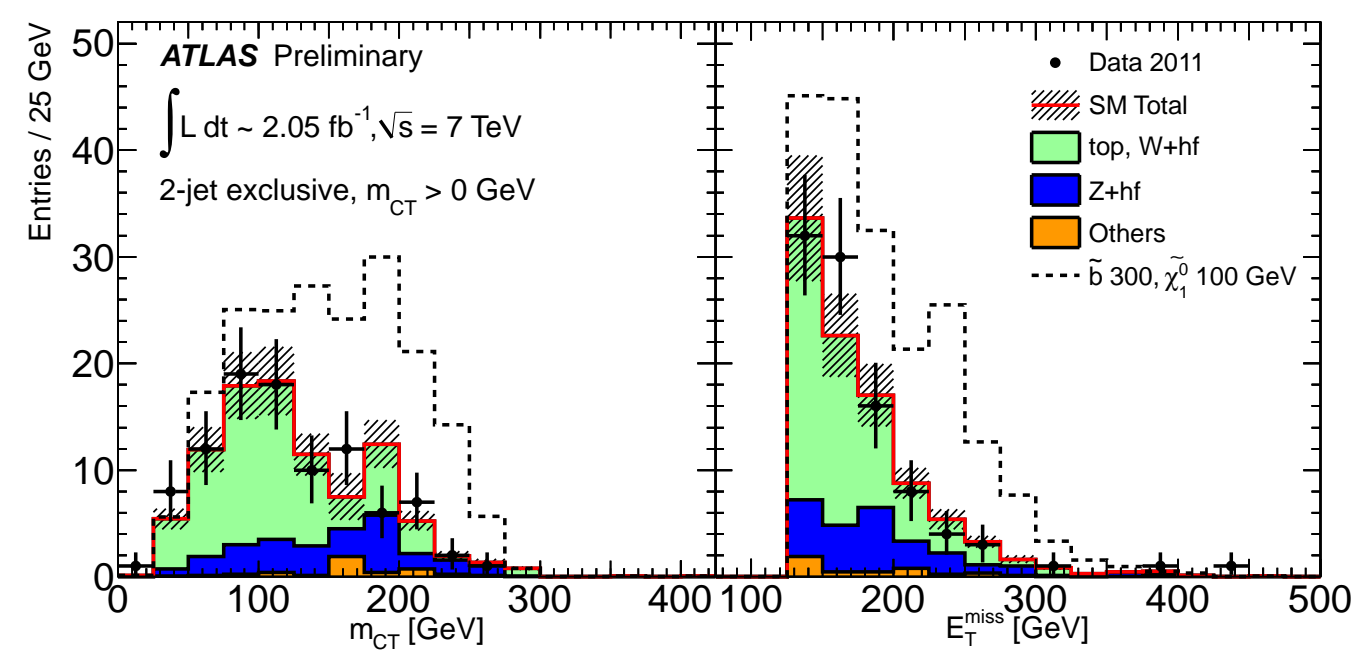

Figure 9: The $\mathrm{m}_{\mathrm{CT}}$ and $E_{\mathrm{T}}^{\mathrm{miss}}$ distribution for the sbottom pair production analysis. The full event selection has been applied but the final cut on $\mathrm{m}_{\mathrm{CT}}$.

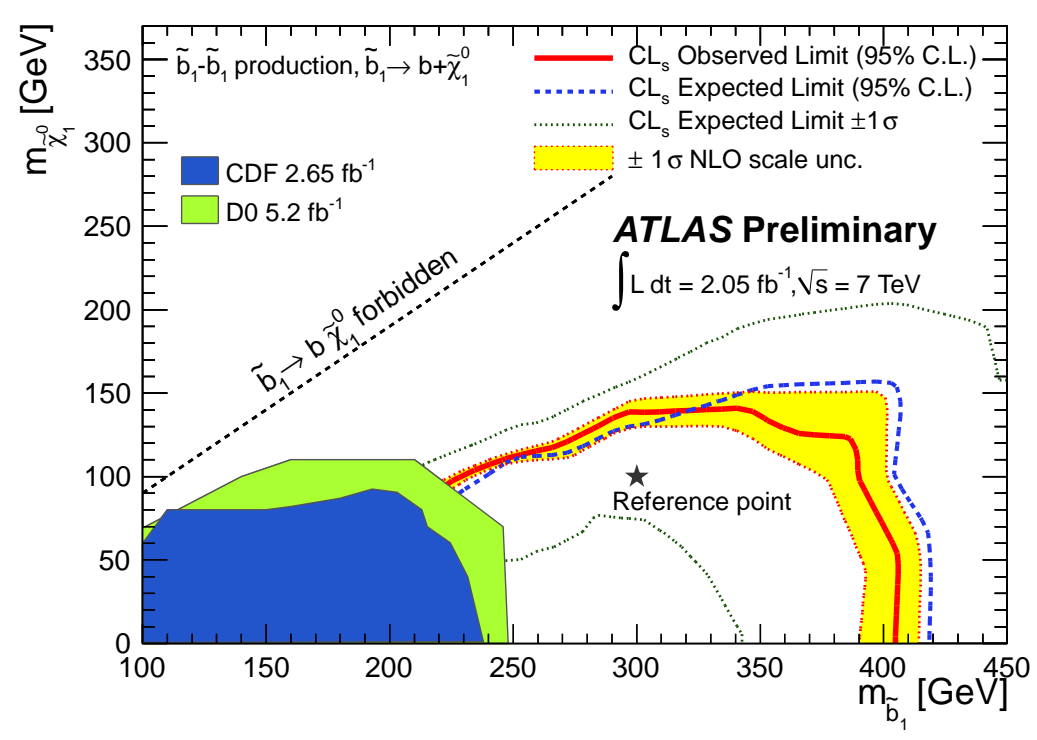

Figure 10: Exclusion limit for the sbottom pair production analysis in the $\tilde{b}_{1}-\tilde{\chi}_{1}^{0}$ mass plane. The decay $\tilde{b}_{1} \rightarrow$ $b \tilde{\chi}_{1}^{0}$ is assumed for both sbottoms. The yellow band takes into account renormalisation and factorisation scale uncertainties for the signal.

\section{R-Parity violating searches}

Two types of R-parity violating searches are presented. One looking for the signature of an Rparity violating $\tilde{v}_{\tau}$ decay (section 3.1) and one looking for long-lived SUSY particles (section 3.2)

\subsection{Searches for a resonance in the $e \mu$ mass spectrum.}

This analysis is scanning the $e \mu$ mass spectrum for a resonance [14]. It is sensitive to the 

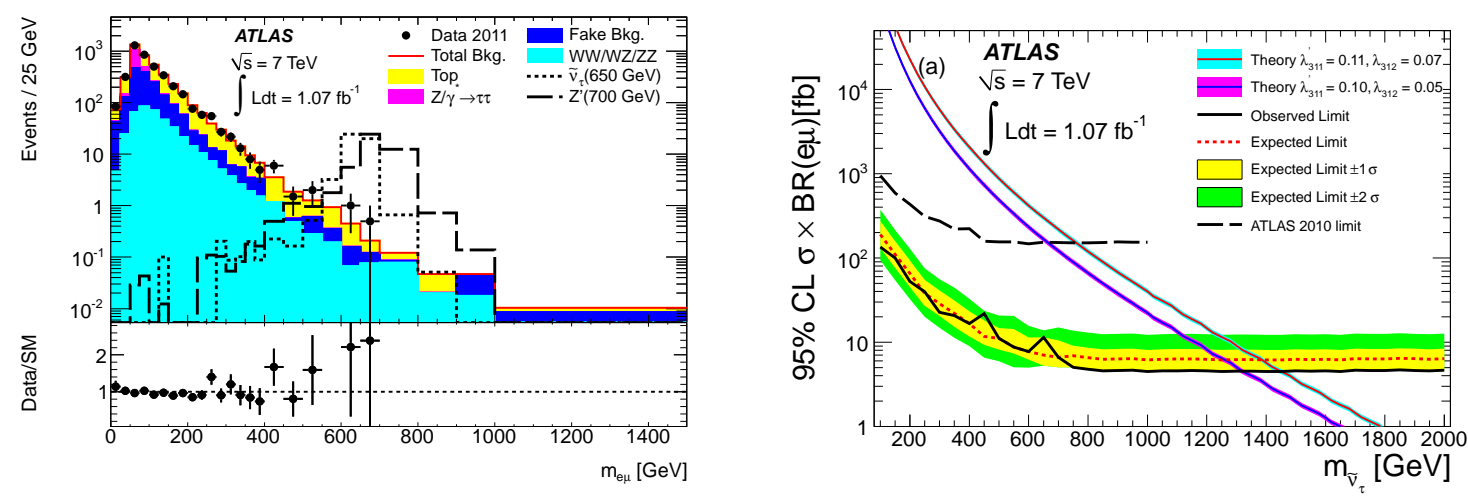

Figure 11: Distribution of the $e \mu$ mass for the resonance search (left). Exclusion limit for the $\tilde{v}_{\tau} \rightarrow e \mu$ cross-section times branching ratio as a function of the tau-sneutrino mass (right). The cross-sections for two theoretical models with different couplings $\lambda_{i j k}$ of the R-parity violating Lagrangian is also shown. Only $\lambda_{311}^{\prime}\left(\tilde{v}_{\tau} \rightarrow d d\right)$ and $\lambda_{312}\left(\tilde{v}_{\tau} \rightarrow e \mu\right)$ are non-zero.

tau-sneutrino decay $\tilde{v}_{\tau} \rightarrow e \mu$, but also for other models such as the existence of a $Z^{\prime}$.

Events with one electron and one muon with opposite sign are selected $\left(p_{\mathrm{T}}>25 \mathrm{GeV}\right)$. The $e \mu$ mass distribution is shown in Fig. 11 (left). No evidence for a resonance is found and the limit on the cross-section times branching ratio for the $\tilde{v}_{\tau} \rightarrow e \mu$ decay is shown in Fig. 11 (right) as a function of the sneutrino mass.

\subsection{Searches for a displaced vertex}

The decay of long-living SUSY particles could form a displaced vertex with a high mass. The search looking for those signatures [15] selects events, where a secondary vertex is present and at least $4 \mathrm{~mm}$ away from the primary vertex. This analysis requires that a muon with high momentum $\left(p_{\mathrm{T}}>45 \mathrm{GeV}\right)$ is associated to the vertex. Furthermore the vertex must have at least four tracks and its mass must be above $10 \mathrm{GeV}$.

The main background comes from the hadronic interaction of primary tracks with the detector material. To reduce it, the position of the vertex is vetoed in high density material regions. Less than 0.03 background events are expected after the event selection.

No data event has been observed and the limit derived for simplified models is shown in Fig. 12. The models are targeting a long-living $\tilde{\chi}_{1}^{0}$ which is produced in $\tilde{q} \tilde{q}$ events via $\tilde{q} \rightarrow \tilde{\chi}_{1}^{0} q$. The limits on the cross-section times branching ratio are given for different squark and neutralino masses as a function of the $\tilde{\chi}_{1}^{0}$-lifetime.

\section{Summary and conclusions}

A broad range of searches for SUSY at ATLAS with different final states have been presented. No excess over the SM expectation was found for $\mathscr{L} \sim 1 \mathrm{fb}^{-1}$ of data. A summary of exclusion limits by the different searches is shown in Fig. 13.

Although no evidence for SUSY was found, more data is available for evaluation and even more data will be recorded in 2012. Besides the optimisation of the existing searches new 


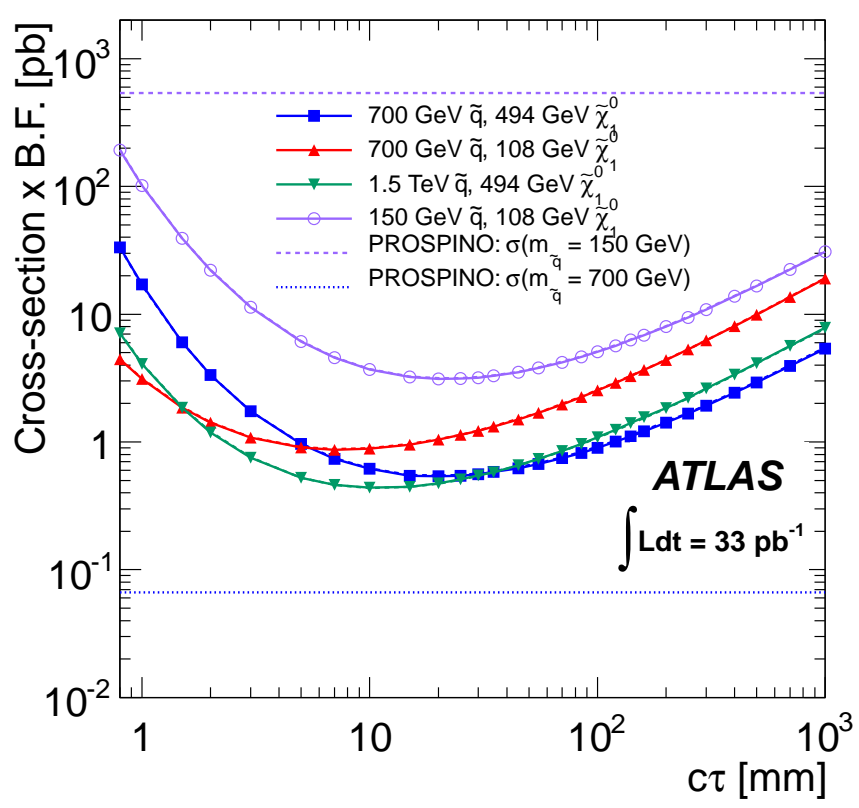

Figure 12: Limit on the production cross-section times branching ratio of $\tilde{q} \tilde{q}$ events decaying to a long living $\tilde{\chi}_{1}^{0}$. The limit is given for various squark and neutralino masses as a function of the $\tilde{\chi}_{1}^{0}$ lifetime times speed of light.

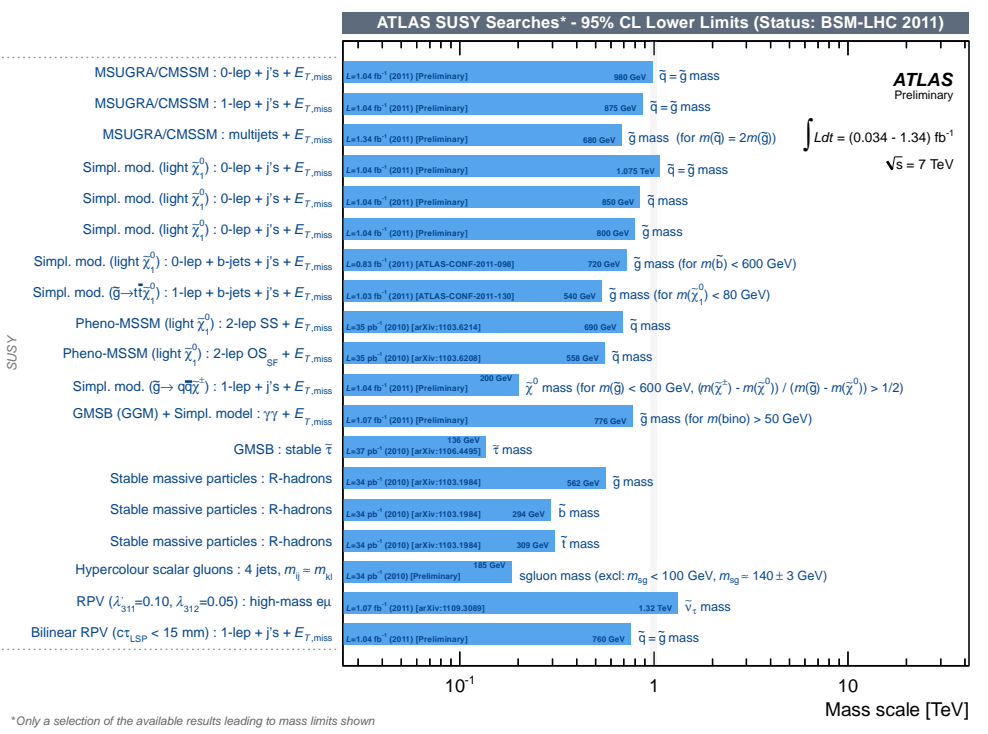

Figure 13: Summary of the mass limits obtained by ATLAS SUSY searches with $0.03 \mathrm{fb}^{-1}<\mathscr{L}<1.3 \mathrm{fb}^{-1}$ and $\sqrt{s}=7 \mathrm{TeV}$.

channels with lower sensitivity can be analysed, such as the direct search for third generation squarks and sleptons and the weak production of SUSY particles. 


\section{References}

[1] Yu. A. Golfand and E. P. Likhtman, JETP Lett. 13, 323 (1971); A. Neveu and J. H. Schwartz, Phys. Rev. D 4, 1109 (1971); P. Ramond, Phys. Rev. D 3, 2415 (1971); D. V. Volkov and V. P. Akulov, Phys. Lett. B 46, 109 (1973); J. Wess and B. Zumino, Nucl. Phys. B 70, 39 (1974); G. R. Farrar and P. Fayet, Phys. Lett. B 76, 575 (1978).

[2] S. P. Martin, A Supersymmetry Primer, arXiv:hep-ph/9709356v4, 2006.

[3] ATLAS Collab., JINST 3, S08003 (2008).

[4] ATLAS Collab., Search for squarks and gluinos using final states with jets and missing transverse momentum with the ATLAS detector in $\sqrt{s}=7$ TeV proton-proton collisions, arXiv:1109.6572v1, 2011.

[5] ATLAS Collab., Search for supersymmetry with jets and missing transverse momentum: Additional model interpretations, ATLAS-CONF-2011-155, CERN, Geneva, 2011.

[6] ATLAS Collab., JHEP 11, 099 (2011).

[7] ATLAS Collab., Search for supersymmetry in final states with jets, missing transverse momentum and one isolated lepton in $\sqrt{s}=7$ TeV pp collisions using $1 \mathrm{fb}^{-1}$ of ATLAS data, arXiv:1109.6606, 2011.

[8] ATLAS Collab., Searches for supersymmetry with the ATLAS detector using final states with two leptons and missing transverse momentum in $\sqrt{s}=7$ TeV proton-proton collisions, arXiv:1110.6189, 2010.

[9] ATLAS Collab., Constraining the gauge-mediated Supersymmetry breaking model in final states with two leptons, jets and missing transverse momentum with the ATLAS experiment at $\sqrt{s}=7 \mathrm{TeV}$, ATLAS-CONF-2011-156, CERN, Geneva, 2011.

[10] ATLAS Collab., Search for supersymmetry in pp collisions at $\sqrt{s}=7 \mathrm{TeV}$ in final states with missing transverse momentum, $b$-jets and no leptons with the ATLAS detector, ATLAS-CONF-2011-098, CERN, Geneva, 2011.

[11] ATLAS Collab., Search for supersymmetry in pp collisions at $\sqrt{s}=7$ TeV in final states with missing transverse momentum, $b$-jets and one lepton with the ATLAS detector, ATLAS-CONF-2011-130, CERN, Geneva, 2011.

[12] ATLAS Collab., Search for scalar bottom pair production with the ATLAS detector in pp Collisions at $\sqrt{s}=7 \mathrm{TeV}$, CERN-PH-EP-2011-195, CERN, Geneva, 2011.

[13] G. Polesello and D. Tovey, JHEP 03, 030 (2010).

[14] ATLAS Collab., Eur. Phys. J. C 71, 1809 (2011).

[15] ATLAS Collab., Phys. Lett. B 707, 478 (2012). 